\title{
Staphylococcus warneri: Skin Commensal and a Rare Cause of Urinary Tract Infection
}

\author{
Aparna Kanuparthy ${ }^{1}$, Tejo Challa ${ }^{1}$, Sreenath Meegada ${ }^{1}$, Suman Siddamreddy ${ }^{2}$, Vijayadershan Muppidi ${ }^{3}$ \\ 1. Internal Medicine, University of Texas Health Science Center/Christus Good Shepherd Medical Center, Longview, \\ USA 2. Internal Medicine, Baptist Health Medical Center, Little Rock, USA 3. Internal Medicine, Indiana University \\ Health, Indianapolis, USA
}

Corresponding author: Tejo Challa, tejo.challa@christushealth.org

\begin{abstract}
Coagulase negative Staphylococci often grow in cultures and form one of the most abundant flora among skin microbiome. It is important and challenging to identify and treat clinically significant infections caused by these organisms. Prosthetic devices, catheters and conditions causing immunocompromised states are the risk factors for such infections. We describe a case of clinically significant and symptomatic urinary tract infection (UTI) in a 65 -year-old man with liver cirrhosis caused by Staphylococcus warneri which forms $<1 \%$ of Staphylococcal skin flora. He was treated successfully with fluoroquinolone antibiotic based on culture results. It is important to understand potential of this organism to cause serious infections which warrant culture-directed antibiotic therapy.
\end{abstract}

Categories: Internal Medicine, Infectious Disease, Nephrology

Keywords: staphylococcus warneri, uti, skin commensal, liver disease

\section{Introduction}

Coagulase-negative staphylococci are skin commensals [1-4]. They are often considered as contaminants in clinical specimens. These organisms present a clinical challenge in differentiating contamination from clinically significant infection when they grow in cultures. There has been increased recognition of the clinical relevance of the presence of these organisms in cultures. The risk factors for clinically significant infections with these organisms include immunosuppression, prosthetic devices, and intravascular catheters. Staphylococcus saprophyticus has been recognized as the most common cause of coagulasenegative urinary tract infection (UTI). Staphylococcus warneri is a rare cause of UTI [3]. We describe the case of Staphylococcus warneri UTI in patient with liver cirrhosis resistant to beta-lactam antibiotics. Liver cirrhosis, irrespective of the cause, is known to cause immunocompromised state [5]. Cirrhosis impairs several aspects of immune response related to both innate and adaptive immunity $[6,7]$.

Received 04/16/2020 Review began 05/18/2020 Review ended 05/19/2020 Published 05/28/2020

(c) Copyright 2020

Kanuparthy et al. This is an open access article distributed under the terms of the Creative Commons Attribution License CC-BY 4.0., which permits unrestricted use, distribution, and reproduction in any medium, provided the original author and source are credited.

\section{Case Presentation}

The patient is a 65 -year-old male with a history of cirrhosis of liver due to alcohol abuse, atrial fibrillation, hypertension, obstructive sleep apnea, hyperlipidemia, and history of deep venous thrombosis (DVT). He presented to the hospital with generalized weakness, pain with urination, and urinary frequency with a need to void every 15 minutes. This was associated with episodes of incontinence. The patient did not report any fevers or chills, had nausea, vomiting. He had intermittent episodes of a small amount of blood in the stool. He continues to drink heavily and has a history of smoking. The patient was intoxicated with alcohol at presentation. He did not have any history of urological problems or a history of previous urinary tract infections requiring treatment. He has no significant family history of liver disease or immunological disorders.

On physical examination, the patient was afebrile with normal blood pressure, pulse, and oxygen saturation. Heart and lung sounds were normal; the examination of the abdomen was significant only for mild epigastric tenderness, no significant costo-vertebral angle tenderness. The patient was euvolemic without significant ascites, peripheral edema. The patient had pancytopenia, urine toxicology screen was positive for alcohol, and cannabis, and urinalysis showed amber-colored urine with leukocytes, positive leukocyte esterase, and negative nitrite (Tables 1, 2). 


\section{Cureus}

\begin{tabular}{|l|l|}
\hline White blood count & $3200 / \mathrm{mm}^{3}$ \\
\hline Red blood count & $3.44 \mathrm{million} / \mathrm{mm}^{3}$ \\
Hemoglobin & $11 \mathrm{gram} / \mathrm{dL}$ \\
Hematocrit & $31.8 \%$ \\
Platelet count & $39,000 / \mathrm{mm}^{3}$ \\
Segmented neutrophils & $77 \%$ \\
Band neutrophils & $1 \%$ \\
\hline Lymphocytes & $19 \%$
\end{tabular}

TABLE 1: Complete blood cell count showing pancytopenia secondary to chronic liver disease

\begin{tabular}{|l|l|}
\hline \hline Urine color & Amber \\
\hline Urine pH & 6.0 \\
\hline Urine protein & $30 \mathrm{mg} / \mathrm{dL}$ \\
\hline Urine blood & Moderate \\
\hline Urine bilirubin & Small \\
\hline Urine leukocyte esterase & Positive \\
\hline Urine nitrite & Negative \\
\hline Urine red blood cell count & 3 cells/high power field \\
\hline Urine white blood cell count & 161 cells/high power field \\
\hline
\end{tabular}

TABLE 2: Urine analysis showing elevated white blood cell count, positive leukocyte esterase

The patient was admitted to the hospital, monitored for alcohol withdrawal, gastro-intestinal bleeding, and was started on empiric treatment with ceftriaxone for urinary tract infection. There was no recurrence of rectal bleed during the hospital course, and the patient did require benzodiazepines for alcohol withdrawal. The patient's MELD score was 17. Urine cultures were sent. Cultures grew coagulase-negative staphylococci. Subsequently, the organism was identified as Staphylococcus warneri with 75,000-100,000 colony forming units $/ \mathrm{ml}$ on culture results. The organism is resistant to Penicillins and was beta-lactamase producing (Table 3). 


\section{Cureus}

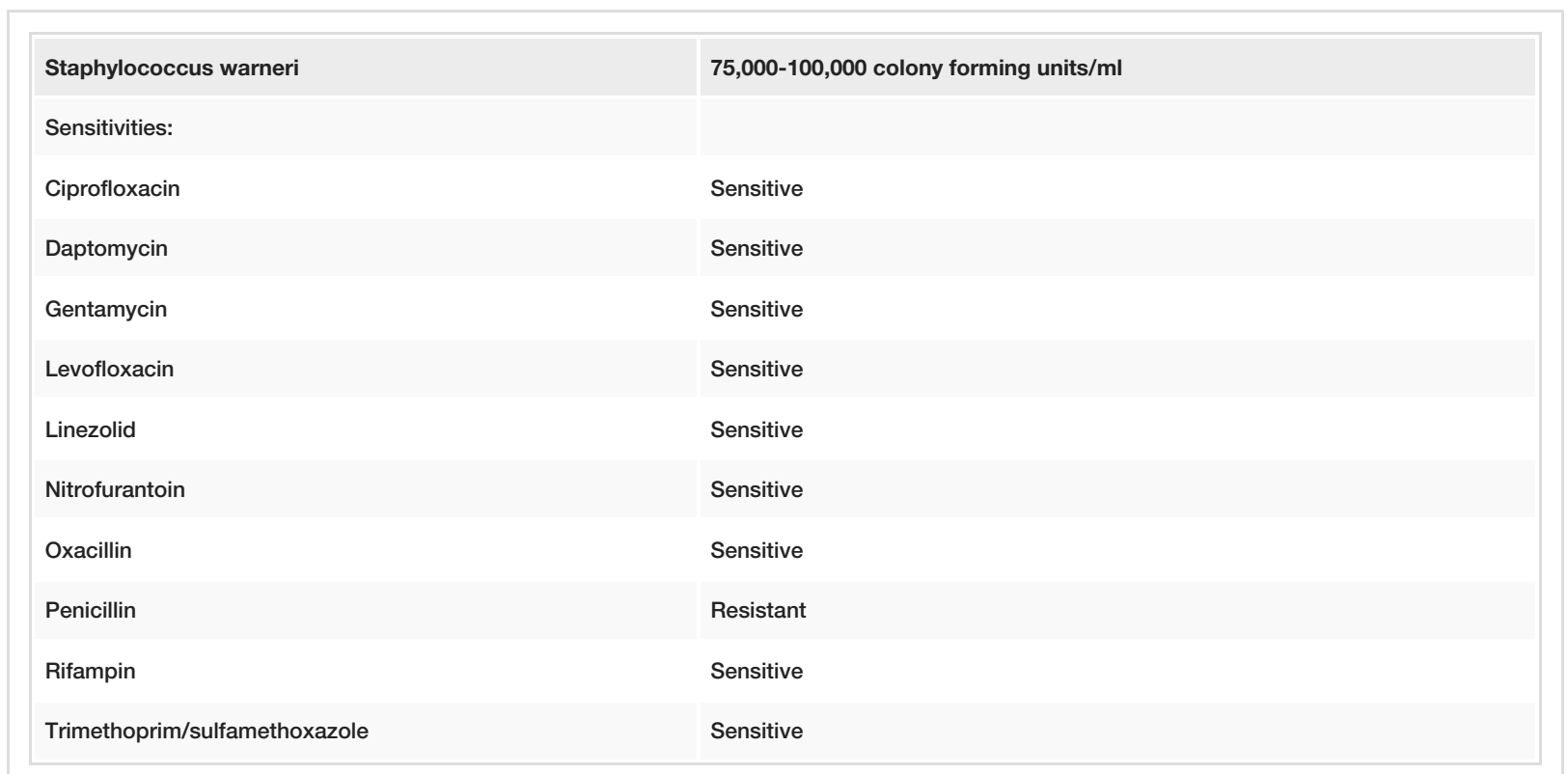

TABLE 3: Urine culture showing organism and sensitivities

It was sensitive to fluoroquinolone antibiotics and Bactrim. The patient was started on levofloxacin with improvement. The patient did have an episode of bleeding from the penis, which required a urology evaluation. Urologist recommended culture-directed antibiotics and possible cystoscopy if there is a recurrence of hematuria. The patient's dysuria and urinary frequency resolved. There was no hematuria or recurrence of bleeding from the penis. The patient was discharged home on oral levofloxacin with scheduled outpatient follow-up. The patient was encouraged to quit drinking alcohol. He was given alcohol cessation counseling and resources for alcohol cessation.

\section{Discussion}

It is important to recognize the role of coagulase-negative Staphylococci in causing UTIs, among other serious infections. Their role in the pathogenesis of such infections has drawn more attention in the past several years. Staphylococcus saprophyticus, S. epidermidis, S. haemolyticus and S. warneri account for most of the responsible organisms [8]. S. saprophyticus is the most common cause of UTIs. S. warneri is catalase positive, oxidase negative, coagulase-negative organism, which is a commensal on skin flora. It constitutes $<1 \%$ of skin Staphylococcus flora [9]. It is a rare cause of sepsis, and an immunocompromised state is a predisposing factor for such infections [10]. There are reported cases of catheter-related bacteremia, endocarditis, multiple abscesses, septic arthritis caused by Staphylococcus warneri [10,11]. Isolates from these infections are generally resistant to Penicillins, with beta-lactamase activity and show susceptibility to other antibiotics with gram-positive activity. Infections due to these organisms were successfully treated with fluoroquinolones, among other antibiotics [12].

\section{Conclusions}

Coagulase-negative Staphylococci are frequently isolated from urinary specimens. Absence of symptoms, pyuria, and the presence of other skin and urethral flora help exclude clinically significant infections. Urine samples, in many cases, may show growth of $<100,000$ colony forming units (CFU). Appropriate setting based on clinical symptoms, presence of other commensal skin, and urethral organisms in urine help determine the clinical significance of the culture results. Staphylococcus warneri, which constitutes a tiny part of skin flora, may cause significant infections. Based on the clinical picture and above discussed criteria, clinically significant infections should be treated based on culture results. Non-beta-lactam antibiotics with gram positive activity can successfully treat these infections based on the data available so far.

\section{Additional Information}

Disclosures

Human subjects: Consent was obtained by all participants in this study. Conflicts of interest: In compliance with the ICMJE uniform disclosure form, all authors declare the following: Payment/services info: All authors have declared that no financial support was received from any organization for the submitted work. Financial relationships: All authors have declared that they have no financial relationships at present or within the previous three years with any organizations that might have an interest in the submitted work. Other relationships: All authors have declared that there are no other 
relationships or activities that could appear to have influenced the submitted work.

\section{References}

1. Roth RR, James WD: Microbial ecology of the skin. Annu Rev Microbiol. 1988, 42:441-464. 10.1146/annurev.mi.42.100188.002301

2. Grice EA, Kong HH, Conlan S, et al.: Topographical and temporal diversity of the human skin microbiome. Science. 2009, 324:1190-1192. 10.1126/science.1171700

3. Grice EA, Segre JA: The skin microbiome. Nat Rev Microbiol. 2011, 9:244-253. 10.1038/nrmicro2537

4. Becker K, Heilmann C, Peters G: Coagulase-negative staphylococci. Clin Microbiol Rev. 2014, 27:870-926. 10.1128/CMR.00109-13

5. Dimitriadi D, Charitidou C, Charvalos E: Urinary tract infection due to beta-lactams-resistant Staphylococcus warneri: a case report. Int Arabic J Antimicrob Agents. 2015, 4:4. 10.3823/760

6. Tuchendler E, Tuchendler PK, Madej G: Immunodeficiency caused by cirrhosis. Clin Exp Hepatol. 2018, 4:158-164. 10.5114/ceh.2018.78119

7. Leighton PM, Little JA: Identification of coagulase-negative Staphylococci isolated from urinary tract infections. Am J Clin Pathol. 1986, 85:92-95. 10.1093/ajcp/85.1.92

8. Hashmi A, Abdullah FE, Abdullah NE, Kazmi SU: Species identification and antibiotic susceptibilities of coagulase-negative staphylococci isolated from urinary tract infection specimens. J Coll Physicians Surg Pak. 2016, 26:581-584.

9. Kloos W: Natural populations of the genus Staphylococcus . Ann Rev Microbial. 1980, 34:559-592. 10.1146/annurev.mi.34.100180.003015

10. Kloos WE, Schleifer KH: Isolation and characterization of Staphylococci from human skin II. Descriptions of four new species: Staphylococcus warneri, Staphylococcus capitis, Staphylococcus hominis, and Staphylococcus simulans. Int J Syst Bacteriol. 1975, 25:62-79. 10.1099/00207713-25-1-62

11. Ivić I, Karanović J, Pavičić-Ivelja M: Sepsis with multiple abscesses caused by Staphylococcus warneri: a case report. Cent Eur J Med. 2012, 8:45-47. 10.2478/s11536-012-0066-0

12. Legius B, van Landuyt K, Verschueren P, Westhovens R: Septic arthritis due to Staphylococcus warneri: a diagnostic challenge. Open Rheumatol J. 2012, 6:310-311. 10.2174/1874312901206010310 